AIAA-2001-3641

\title{
Addressing EO-1 Spacecraft Pulsed Plasma Thruster EMI Concerns
}

\author{
C.M. Zakrzwski \\ Guidance. Navigation, and Control Center \\ NASA-Goddard Space Flight Center \\ Greenbelt, MD 20771 \\ Mitch Davis \\ NASA-Goddard Space Flight Center \\ Greenbelt. MD 20771 \\ Charles Sarmiento \\ NASA John H. Glenn Research Center \\ Cleveland, $\mathrm{OH}$
}

\begin{abstract}
The Pulsed Plasma Thruster (PPT) Experiment on the Earth Observing One (EO-1) spacecraft has been designed to demonstrate the capability of a new generation PPT to perform spacecraft attitude control. Results from PPT unit level radiated electromagnetic interference (EMI) tests led to concerns about potential interference problems with other spacecraft subsystems. Initial plans to address these concerns included firing the PPT at the spacecraft level both in atmosphere, with special ground support equipment, and in vacuum. During the spacecraft level tests. additional concerns where raised about potential harm to the Advanced Land Imager (ALI). The inadequacy of standard radiated emission test protocol to address pulsed electromagnetic discharges and the lack of resources required to perform compatibility tests between the PPT and an ALI test unit led to changes in the spacecraft level validation plan. An EMI shield box for the PPT was constructed and validated for spacecraft level ambient testing. Spacecraft level vacuum tests of the PPT were deleted. Implementation of the shield box allowed for successful spacecraft level testing of the PPT while eliminating any risk to the ALI. The ALI demonstration will precede the PPT demonstration to eliminate any possible risk of damage of ALI from PPT operation.
\end{abstract}

Introduction

A Pulsed Plasma Thruster (PPT) is being flown as a technology demonstration experiment on the Earth Observing 1 (EO-1) mission. The EO- 1 mission is managed by the NASA Goddard Space Flight Center (GSFC) for the New Millennium Program (NMP). The EO-1 PPT is the first flight PPT developed in over 10 years and incorporates significant improvements over the previous generation of PPTs. The EO-I PPT experiment was developed by NASA GSFC. NASA Glenn Research (GRC), General Dynamics Ordnance and Tactical Systems (formerly Primex Aerospace Corporation), and Swales Aerospace. The EO-1 PPT flight experiment will demonstrate the ability of this new generation of PPT to provide precision attitude control and confirm benign interaction with other spacecraft subsystems and instruments. On EO-1, the PPT will be tested as a "replacement" for one of the reaction wheels. Because the EO-1 PPT experiment was classified as a category III experiment (nonessential to primary instrument validation mission) it was sufficient for validation purposes to design the experiment such that a single PPT unit would control a single spacecraft axis. For ease of integrating the PPT into the spacecraft. the pitch axis was chosen and the EO-1 PPT was designed with two opposing thruster electrodes in the positive and negative pitch direction.

\section{Background}

\section{PPT Description}

The EO-1 PPT is a small. self-contained electromagnetic propulsion system that utilizes solid Teflon propellant. It can deliver high specific impulses $(650-1400 \mathrm{sec})$. very fine impulse bits $(90-860$ 
$\mu N-s)$ at lou power levels $12-70 \mathrm{~W}$. The detailed performance characteristics and development of the EO-1 PPT are described in previous publications Figure 1 shows the PPT mounted to the spacecraft while the spaceraft is attached to the bunch vehicle

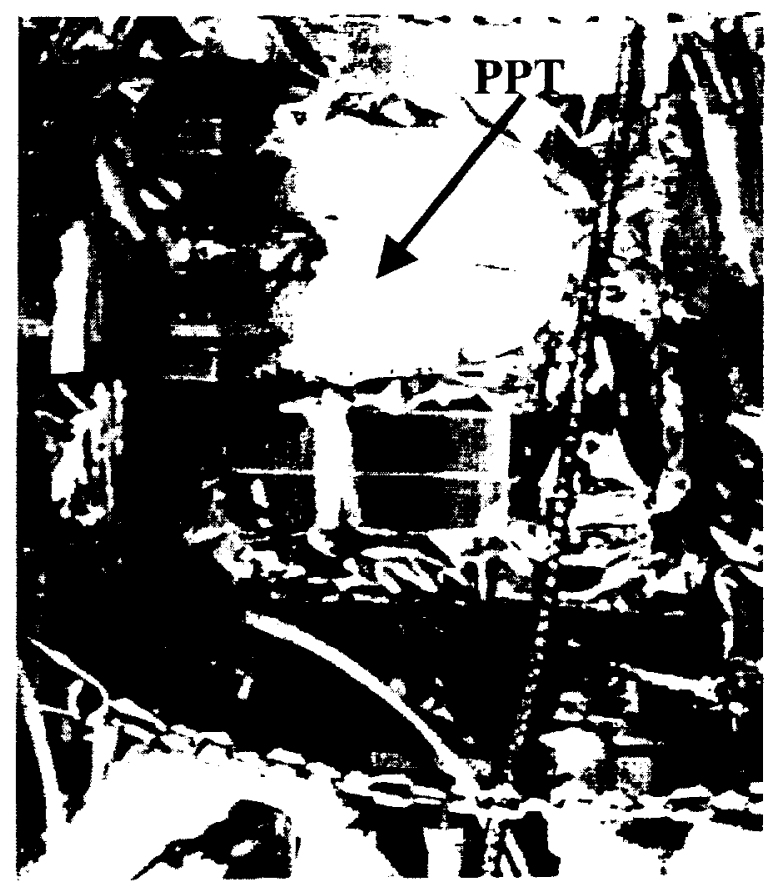

Figure 1: EO-1 spacecraft with PPT

PPTs can offer significant mass saving benefits to spacecraft by replacing the combinations of reaction wheels, torque rods. and chemical thrusters. ${ }^{45}$ PPTs have the advantage of being simple to integrate to spacecraft because of the limited mechanical mounting hardware and electrical requirements. PPTs also eliminate safety and component manifold complexities associated with fluid propellant propulsion systems

The operation of the PPT is inherently simple. Referring to Figure 2. the main capacitor is initially charged to the desired level and then discharged across the face of a Teflon fuel bar. Two fuel bars are located between separate and opposing electrode pairs to provide thrust in the positive and negative $Z$-axis directions (the PPT location with respect to the center of mass results in $+/$ - pitch torquel. The discharge of the main capacitor occurs when the spark plug on the desired electrode palir is commanded to fire. A minute amount of charged particles is ablated into the electrode gap when the spark plug is fired. These charged particles provide a conductance path that initiates the main capacilon discharge across the gap. The main capacitor discharge ablates a small amount of Tethon A small pereentige of the Teflon is ionized fo form plasma. A Lorentz force accelerates the plasma producing thrust. Charged particle to neutral collisions. and pressure forces from resistive heating produce additional acceleration of the neutral charged. ablated Teflon.

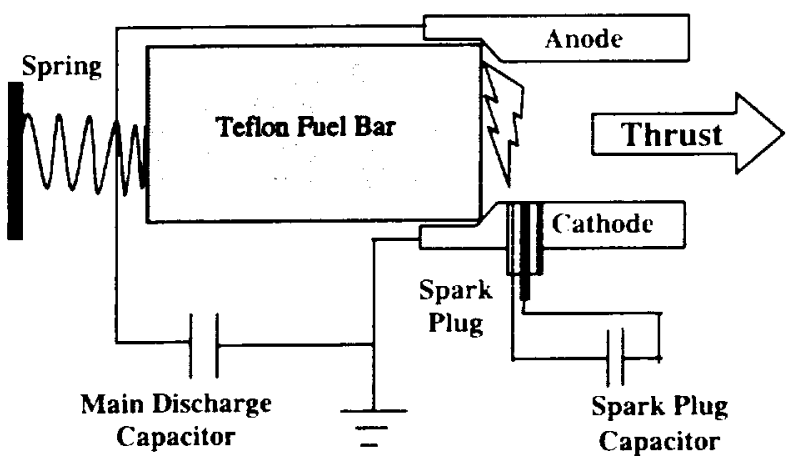

Figure 2: PPT Diagram

\section{$\underline{\text { EO-1 Mission }}$}

The EO- 1 mission is managed by Goddard Space Flight Center. It is the first Earth Observing mission for the NASA New Millennium Program (NMP) ${ }^{3}$. The purpose of the mission is to validate revolutionary technologies contributing to the reduction in cost and increase in capabilities for future land imaging missions. The spacecraft carries three advanced land imaging instruments, the Advanced Land Imager (ALI), the Atmospheric Corrector, and the Hyperion (Hyperspectral Imager). The spacecraft also carries seven spacecraft bus technologies: the Wideband Advanced Recorder \& Processor (WARP), a deployable light-weight flexible solar array, an advanced $\mathrm{X}$-band phased array, autonomous formation flying software, a fiber optic data bus, a carbon-carbon radiator, and the PPT. A illustration of the EO-1 spacecraft instruments and spacecraft bus technologies is shown in Figure 3.

EO-1 was launched on November 21, 2000 into a 705 $\mathrm{km}$ circular, sun-synchronous orbit at a 98.7-degree inclination. It flies within one minute of the Landsat 7 spacecraft in order to collect identical images for comparison and validation. As of May 11, 2001, a full series of calibration and performance scenes has been collected for all three instruments. Preliminary results of the analyses of the scenes indicate that all three instruments are performing as specified. All spacecraft bus technologies. excluding the PPT. have also been demonstrated successfully. 


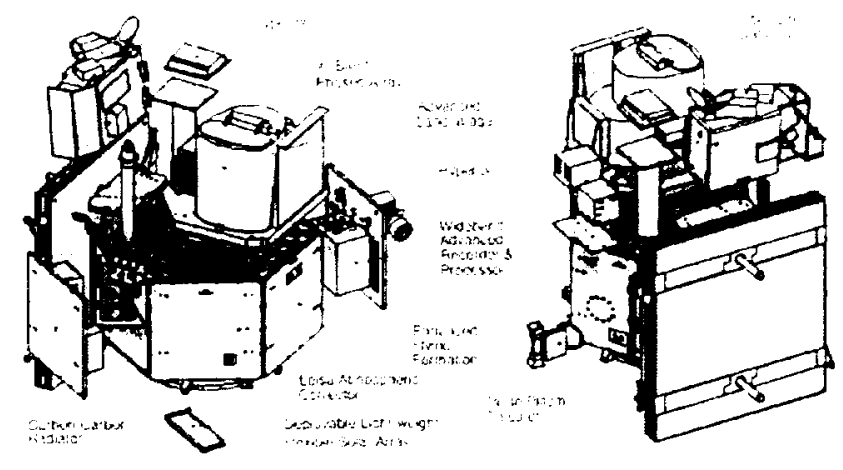

Figure 3: EO-1 Technologies

\section{PPT Flight Experiment}

The PPT technology validation will consist of operating the PPT as a replacement for the pitch wheel in EO-l's attitude control loop. The PPT will provide pitch axis torque after the pitch axis momentum wheel and magnetic torquer bar have been disabled. This substitution will occur in the nadir pointing mission mode of the spacecraft. The spacecraft disturbance forces include solar pressure torques, aerodynamic drag, gravity gradient torques, bearing friction and torques generated by the solar array drive. The solar array drive produces the greatest disturbance and will require the greatest amount of control torque from the PPT.

The PPT will perform pitch axis control for a minimum of three days, and has enough Teflon propellant to operate continuously for approximately thirty days. Images taken from the three scientific instruments during PPT operations will be used to assess what effect, if any, the PPT has on these instruments. Initial operation and testing of the PPT will take place with the spacecraft controlled by all three momentum wheels. Only after PPT on-orbit performance is verified will the PPT be substituted for the pitch axis wheel in the closed-loop system.

\section{EO-1 EMI Requirements and Acceptance Testing}

\section{Requirements and Test Methods}

The EO-1 requirements for Electromagnetic Interference (EMI) compatibility and susceptibility for all spacecraft subsystem were tailored from MIL-STD462 and MIL-STD-46IC. From MIL-STD-461C. the applicable tests for conducted emissions were CE01. CE03. and CEO7 and for radiated emissions were REO1 and RE02. The standard requirements were tailored to accommodate consideration of the spacecraft communications transmitter/receiver. The specified emission levels between 1.770 and $2.300 \mathrm{GHZ}$ were notched. The EMI requirements were not tailored to accommodate any specific requirement from other spacecraft subsystems or instruments.

\section{Acceptance Test Results}

The acceptance-level EMI testing was performed by NASA/GRC'. Because the results from the RE02 radiated emissions tests led to the concern that the emissions may harm the ALI. these results will be discussed in some detail.

To obtain useful data for the RE02 requirements, NASAVGRC had to utilize unique test methods to characterize the PPT. Radiated emissions tests were performed with the PPT firing in a glass vacuum bell jar. A conventional automated frequency sweep was considered inadequate to capture peak emissions levels over the entire frequency range of interest due to the nature of the PPT discharge. The dominant radiation event of the PPT firing is a broadband pulse that lasts for a relatively short time $(<1-2 \mu \mathrm{s})$. The event repeats at the relatively low $1 \mathrm{~Hz}$. PPT frequency. In addition, testing showed a large 10-30 dB shot to shot variation in measured signal amplitude.

An alternative approach to the conventional frequency sweep test was developed by GRC after considering previous PPT experience. including MIT Lincoln Laboratory's LES 6-9 programs, TIP-II, NOVA. AFRPL/Fairchild Millipound PPT program. and NASDA's ETL ETS-IV space test. The test receiver was manually tuned to selected frequencies across the $14 \mathrm{kHz}$ to $18 \mathrm{GHz}$ range. Measurements at these selected frequencies were taken by firing the PPT numerous times in order to account for shot to shot variations. The GRC method gave a more representative characterization of the erratic but dominant broadband emission. Although this method overlooks emissions between the selected test frequencies, the nature of the discharge of the PPT can be best classified as an isentropic broadband emission that is highly unlikely to produce narrow-band emissions. Initial testing verified no clear directionality in the emissions.

Three different antennas were used to cover the RE02 frequency range: an eight band passive rod for $14 \mathrm{kHz}$ to $32 \mathrm{MHz}$ : a hydrid biconical/log periodic dipole for $25 \mathrm{MHz}$ to $2 \mathrm{GHz}$ and a small log periodic dipole for 1 to $18 \mathrm{GHz}$. PPT emissions at test frequencies in the overlap ranges were measured with both antennas to check agreement. 


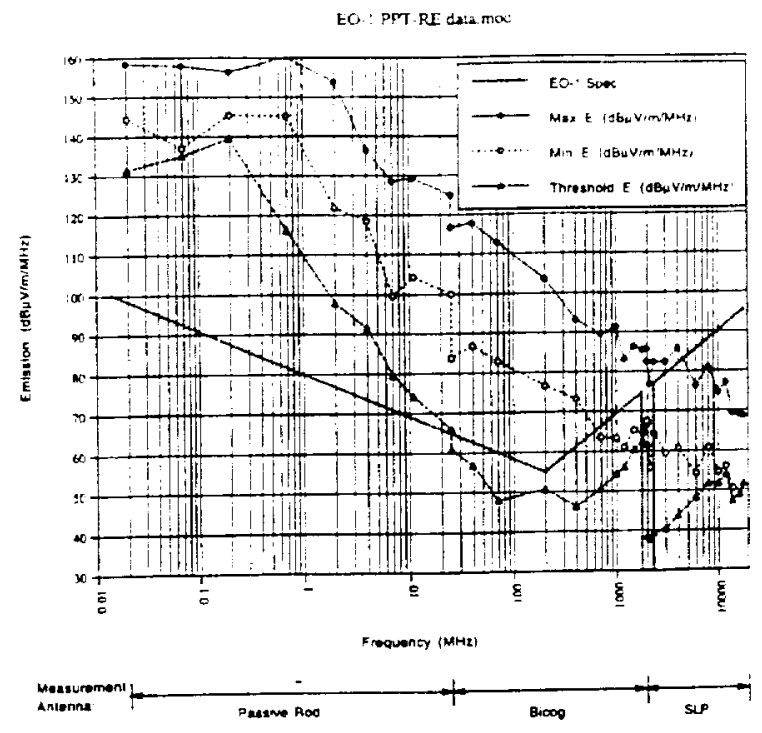

Figure 4: EO-1 RE02 Results

The results of these tests are shown in Figure 4. For each selected frequency the PPT was fired 100 times at its maximum firing energy of $57 \mathrm{~J}$ on the main capacitor. The maximum and minimum emission for each 100 shot group is shown in Figure 4. The values for emissions at frequencies between the selected measurement frequencies are interpolations. The dominant emission for every shot at all tested frequencies was found to be approximately 1 microsecond in duration at the moment of igniter/main discharge firing. The PPT firing frequency fot the test was $1 \mathrm{~Hz}$. It is this peak which is plotted.

As seen in Figure 4, the electric field emissions exceed the spacecraft level requirements by as much as 60 to $80 \mathrm{~dB}$ over a broad range of frequencies. Only above the $2-3 \mathrm{GHz}$ range do the emissions fall at or below the EO-1 specifications. The high emissions at the lower frequencies have been observed in previous PPT programs and are common for this type of electrical discharges. Other electric propulsion devices such as arcjets and Hall thrusters have similar characteristics and have been successfully flown on numerous tlight missions without any detrimental effects to other spacecraft subsystems or instruments. Although the PPT emissions exceeded the specified emission limits in the spacecraft transmitter/receiver frequency range. it was concluded that this would not present a problem due to the extremely short duration of the emission event. Assuming that the PPT did create noise on the transmitter/receiver, at the lower spacecraft house keeping data rate of $2 \mathrm{~kb} / \mathrm{sec}$. the very short emission pulse was estimated not to be long enough to affect a single data bit. At the higher $105 \mathrm{Mb} / \mathrm{sec}$ rate. the number of bits effected by the PPT would be insignificant because of the low $1 \mathrm{~Hz}$ firing frequency.

\section{Initial PPT spacecraft integration tests.}

The original plan to integrate and test the PPT at the spacecraft level included two types of tests: ambient level PPT firings using special ground support equipment (GSE) and vacuum level PPT firings during spacecraft thermal vacuum testing. An additional spacecraft level test, with the PPT firing in a vacuum bell jar, was added prior to mechanical integration of the PPT because of the results of the radiated emissions tests.

\section{Vacuum Bell Jar Test}

The purpose of this test was to ensure that the emissions from the PPT, when discharged in a vacuum, would not cause any spacecraft component anomaly. The glass vacuum bell jar with the PPT was located as close to the spacecraft as physically possible. The PPT was connected to the spacecraft through an extension harness, and the firing commands were issued from spacecraft command subsystem. The reason to perform this test early in the spacecraft I\&T schedule was to allow time for possible changes in the PPT or spacecraft shielding design.

The PPT spacecraft level vacuum bell jar test occurred in December 1998. The spacecraft bus subsystems were electrically integrated and the spacecraft external panels were open. The spacecraft bus components, which would normally be shielded by the external panels on orbit, were directly exposed to the PPT emissions during this test. Offsetting this very conservative EMI test configuration was the less than conservative physical location of the PPT. The PPT could only be located within approximately one meter of its flight mounting position on the spacecraft because of the size of the vacuum bell jar. None of the EO- 1 instruments were integrated on the spacecraft for this test.

Prior to firing the PPT in the vacuum. the PPT was electrically mated to the spacecraft and fired in ambient air utilizing the PPT GSE. The PPT GSE allowed connections to be made from the flight electrodes to a resistive load box. This enabled the main capacitor to be charged and then discharged through the load-box without causing a potentially damaging in-air electrical arc. Included as part of this GSE. electrical contacts were located over the PPT spark plugs and were connected to a load-box triggering unit. When the spark plugs were commanded to fire. the GSE would trigger a discharge of the main capacitor through the load box by a signal from the triggering unit. 
The PPT was fired by spacecraft commands approximately 50 times for both the ambient and vacuum test configurations. During the firings, the spacecraft was powered up with all relevant subsystems functioning. The sequence of 50 shots was divided evenly between charging the PPT to its maximum and to its minimum flight charge capacity. No spacecraft anomalies were observed during any of the PPT firings. Additionally, no spacecraft anomalies occurring after the testing could be attributed to PPT operation.

\section{Post mechanical integration test}

After the PPT was mechanically mated to the spacecraft in April 1999. electrical functional tests where performed using the PPT GSE. At this point in the spacecraft integration schedule the Advanced Land Imaginer (ALI) had already been integrated to the spacecraft. Once again the PPT was fired over 50 times with half the firings at the minimum and half at the maximum charge level. During these tests, the spacecraft was powered on but the ALI was not in operation. The PPT functioned nominally and no anomalies attributable to the PPT were observed in other subsystems

\section{Thermal vacuum spacecraft compatibility test}

Test firing the PPT during spacecraft level thermal vacuum testing had been planned to fully evaluate the EMI compatibility of the PPT with the entire spacecraft. Because of the difficulty in analytically evaluating EMI effects on a complex spacecraft or developing a high fidelity component level test scheme, testing firing the PPT at the spacecraft level was the most feasible approach to test and ensure compatibility. Initially, the risk of harming other spacecraft systems by taking this approach was assumed to be very low. This method has been used on a number of previous PPT flight programs including LES 6-9 programs and the NOVA $1-3$ programs. The lessons learned from these previous programs on reducing emission effects were incorporated in the design of the EO-I PPT. These include effective isolation. grounding, power and signal filtering. and shielding schemes. During discussion about the possible early on-orbit operation of the PPT, the ALI instrument team raised concerns about the effect PPT emissions could have on the instrument. These concerns led to a reevaluation of the PPT spacecraft level test plan.

\section{Advanced Land Imager Concerns}

\section{Instrument Description}

The Advanced Land Imager was designed to demonstrate comparable or improved spatial and spectral resolution with respect to Landsat-7\%s Enhanced Thermatic Mapper (ETM+) while providing substantial mass. volume, power, and cost savings. The ALI employs novel wide-angle optics and a highly integrated multi-spectral and panchromatic spectrometer. ${ }^{6}$

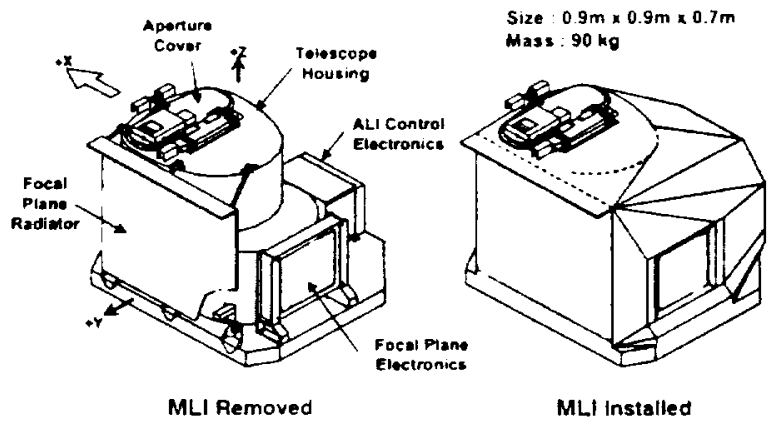

Figure 5: Advanced Land Imager

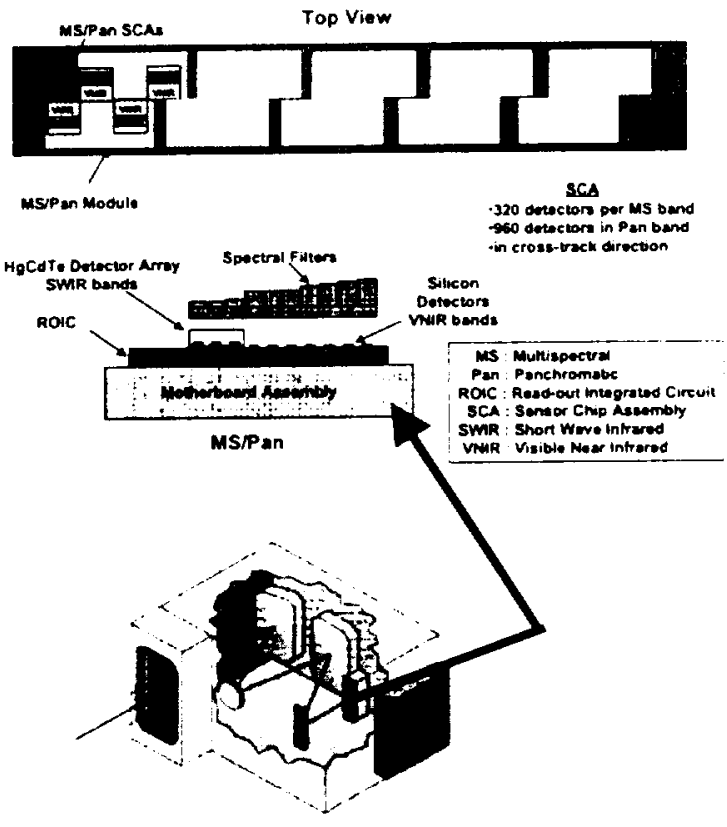

Telescope Housing Schematic

Figure 6: ALI Telescope and Focal Plane Array 
The instrument consists of several components mounted on an instrument pallet as shown in Figure 5. These include the telescope housing. ALI control electronics. Focal Plane Electronics, and Focal Plan Radiator Inside the telescope housing are the optics. Focal Plane Assembly (FPA), and calibration system. The primary concern was the possibility that the PPT radiated emissions could damage the FPA detectors and readout chips. A layout of the FPA is shown in Figure 6.

The Multi-Spectral Panchromatic (MS/Pan) array has 10 spectral bands in the visible. near infrared (VNIR). and short wave infrared (SWIR). The MS/Pan arrays use Silicon-diode VNIR detectors fabricated on the Silicon substrate of a Readout Integrated Circuit (ROIC). The SWIR detectors are Mercury-CadmiumTelluride ( $\mathrm{HgCdTe})$ photo-diodes that are Indium bump-bonded onto the readout circuit that services the VNIR detectors. The very thin 200 angstrom thick silicon-dioxide capacitors and very small semiconductor junctions were identified as potentially the elements of the detector and readout circuits that are the most sensitive to Electro-Static Discharge (ESD) or over-voltage damage. Additionally semi-conductor devices utilized in the FPA electronics and ALI Control Electronics were also believed to be susceptible to ESD damage.

\section{Analysis of Potential Damage to ALI}

An attempt was made to analytically determine the effect of PPT radiated emissions on the ALI. To perform this analysis, it was necessary to predict the maximum strength of the electromagnetic field generated by the PPT at the FPA location and to determine the EMI susceptibility of the ALI FPA

To predict the radiated field strength of the PPT, the elements that contributed to the EMI were evaluated. The PPT has two discharge circuits that contribute to the radiated emissions: the main capacitor circuit and the spark plug circuit. In the PPT RE02 acceptance tests, all peak emission events coincided with the spark plug discharge and initiation of the main capacitor discharge. Previous testing by GRC indicated that in vacuum the spark plug by itself could produce radiated emissions of the same magnitude as those observed in the RE02 tests for the combined spark plug/main capacitor discharges. Because the spark plugs are fired during ambient air testing with the PPT GSE, it was necessary to determine if firing the spark plugs alone in atmosphere could damage the ALl. To address this question GRC pertormed a limited series of REO2 tests measuring the emissions from a breadboard spark plug in air. For the frequency range between $2 \mathrm{MHz}$ and 1 GHz. the spart plug emissions levels were comparable to the emission levels measured from the vacuum firings of the flight PPT and exceeded the EO-1 specifications by as much as $50 \mathrm{~dB}$ in this frequency range. These results indicated that even the ambient discharge of the PPT using the PPT GSE had to be evaluated for potential threat to the ALI.

Data from the required MIL-STD-461 RE02 and RE01 tests on the PPT were not sufficient to determine the strength of the radiated electric and magnetic field at the ALI location. The MIL-STD-461 emission tests were designed for determining if the steady state electromagnetic fields of one electronic device might interfere with the operation of another electronic device meeting a corresponding susceptibility limit. The RE02 test, for example, dates back to the 1953 testing of a World War II-era receiver used on aircraft. ${ }^{7}$ The receiver was connected to its antenna by unshielded wire that was routed through the aircraft adjacent to interference sources. The limits and tests methods used for susceptibility of this antenna are substantially the same as the current RE02 requirements. The MIL-STD461 tests were not designed to characterize the effect of a low repetition rate pulsed discharge on an integrated circuit.

To estimate the fields generated by the PPT, the PPT discharge circuits were modeled as simple antennas whose physical characteristics where determined by tracing the circuit paths to and from the capacitors. Discharge circuit characteristics are given below.

\begin{tabular}{|l|l|l|}
\hline Parameter & Main Capacitor & Spark Plug \\
\hline Discharge Voltage & $1600 \mathrm{~V}$ & $800 \mathrm{~V}$ \\
\hline Max. Current & $27,000 \mathrm{~A}$ & $80 \mathrm{~A}$ \\
\hline Pulse Length & $2.3 \mathrm{micro} \mathrm{sec}$ & 1 micro sec \\
\hline Cap Energy & $56 \mathrm{~J}$ & $0.3 \mathrm{~J}$ \\
\hline
\end{tabular}

Table 1: PPT Discharge Circuits Characteristics

The main capacitor discharge circuit was modeled as a magnetic dipole and the electromagnetic field was estimated using Schelkunoffs solution to Maxwell's Equations. For the main discharge the radiated magnetic field was the primary concern. The magnetic field strength at the focal plane location was estimated to be only 360 nano-Teslas. The spark plug discharge circuit was modeled as a Hertzian dipole. For the spark plug, the radiated electric field was the primary concern. The electric field strength at the focal plane location was estimated to be $100 \mathrm{~V} / \mathrm{m}$. These estimates were obtained by assuming a distance of 0.64 meters from the PPT 10 the FPA with approximately $0.1 \mathrm{~mm}$ aluminum shielding provided by the ALI iclescope housing. A series of simple tests using the breadhoard PPT model were planned to check the validity of this 
model. This test series was not performed because of the complexity of characterizing the circuit paths in the ALI that may be susceptible to EMI-induced voltage.

To relate the field strengths to voltages that would be induced on the ALI circuitry. it was necessary to determine circuit path lengths. loop areas, and capacitances that could pick up the radiated emissions and apply them to the critical components. This task was cancelled because of the prohibitively high cost and effort. and the risks associated with having to open the flight unit to trace wire paths. In addition, there remained questions about the amount of shielding by the ALI enclosures and whether the ALI wiring lengths could pick up radiated emission at certain "resonant" frequencies. The ALI wiring scheme was not specifically designed to provide protection from EMI. For example, the connection between the FPA and FPA electronics box consisted of an unshielded ribbon cable that was fed through open slots in the ALI telescope shield and FPA electronics boxes.

No quantitative data from the instrument supplier was available to define the radiation emission levels that could be tolerated by the ALI FPA or associated electronics. Only the readout chips had a general specification from the subcontractor that indicated that the chips might be damaged at the several tens of volt level. A duplicate or engineering ALI test unit with the FPA and associated electronics was not available for EMI susceptibility testing. An attempt was made to define an equivalent circuit that could be tested with a PPT engineering unit for compatibility. The design fidelity required in the ALI test article proved to be too costly and too time-consuming with respect to accomplishing the primary EO-1 mission objectives.

\section{Resolution of EMI Concerns}

The lack of susceptibility data for the ALI components. the inability to analytically determine emissions effects of the PPT on the ALI with the precision required, and the inability to design a test which would satisfactorily address concerns within the resources available ultimately made it impossible to prove conclusively prior to launch that operation of the PPT would not damage the ALl. Because the ALI is the primary instrument on E0-1, the on-orbit firing of the PPT would be delayed until after completion of the ALI validation.

A conservative approach was also taken to ambient air test firing of the PPT with GSE. Although the PPT was fired with GSE in ambient air while the ALI was integrated to the spacecraft. additional precautions were deemed necessary in light of the results from spark plug only tests discussed above. A non- flight EMI shield that reduced the PPT emissions was installed during ambient air testing. This EMI shield allowed completion of verification tests of PPT functionality while reducing risks to the ALI. The effectiveness of the EMI shield was tested and verified with an engineering breadboard PPT unit prior to installing the shield on the spacecraft. The use of an EMI test shield for spacecraft level tests was proven to be effective on a previous PPT program.

\section{Design and Implementation of PPT I\&T EMI shield}

\section{Shielding design}

The objective of the PPT EMI shield was to provide a significant reduction in the emission levels when the PPT was fired in air with GSE. The shield was required to enclose the PPT flight unit while it was fully integrated to the EO-1 spacecraft. To achieve these requirements, a three-piece design was chosen. Two interlocking base plates were mounted to the top of the PPT flight unit mounting flange. Each plate was fastened to the flange by removing two flight PPT fasteners and using their associated spacecraft panel inserts. Four channels were cut in the base plates to create feed-throughs for four electrical harnesses: two for PPT GSE harnesses, one for a PPT power harness. and one for a PPT command and telemetry harness. The third piece of this enclosure was a six-sided cover that fit over the PPT unit and mounted to the base plates. The box cover was constructed of 1/8 inch aluminum sheet metal welded together with no open seams. The cover was welded to a machined mounting flange. The cover and the base plates where lined with mu-metal in order to reduce radiated magnetic fields.

Initial testing of the PPT enclosure with the BreadBoard (BB) PPT at GRC indicated the need to incorporate an additional shield box for the PPT GSE boxes to reduce emissions originating from solid-state switches. The PPT GSE boxes including the trigger unit, the load box. and a power supply were placed in a commercially available steel box enclosure. All openings on this shield box were sealed with copper tape. The harness between the GSE boxes and the PPT was also shielded with copper tape. As an additional precaution the 120 VAC power cables from the power supply and trigger unit were shielded with copper tape and aluminum foil.

\section{Breadboard PPT Test Unit}

The BB PPT was used to test the effectiveness of the PPT EMI enclosure. The BB PPT utilized the same spark plug and electrode configuration as the flight unit. 


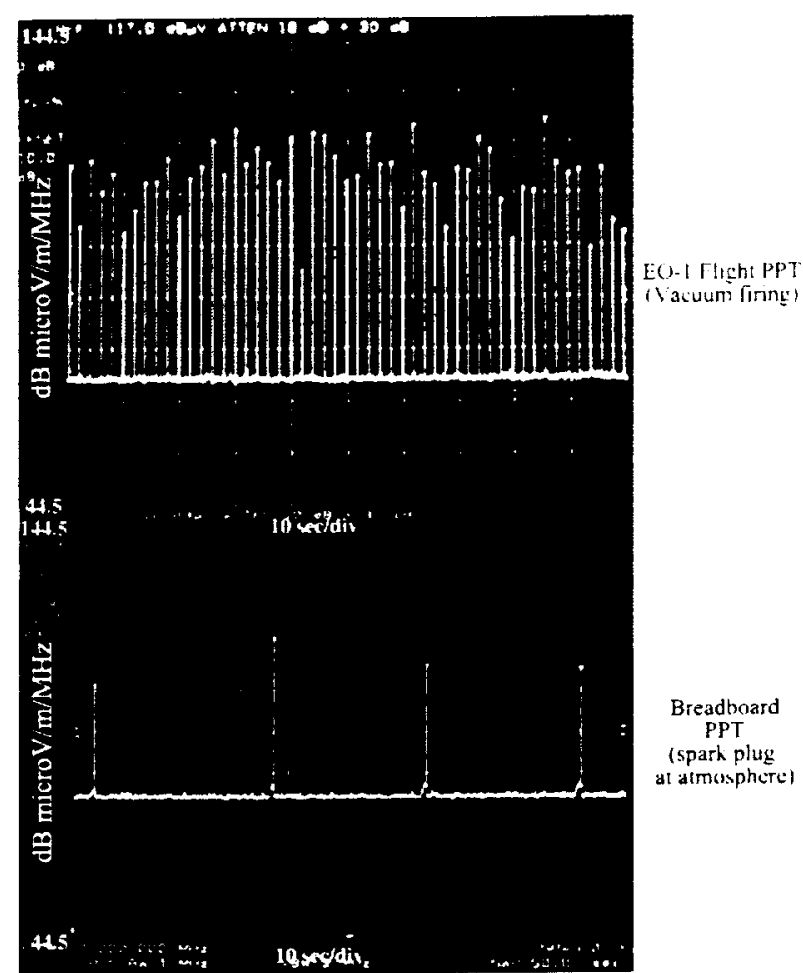

Figure 7. Raw RE02 Data from Flight and Breadboard PPT

The physical layout and some aspects of the BB PPT charging circuit differed from the flight unit. However, both the main capacitor and spark plug discharge circuits were similar enough to have confidence in using the BB PPT as a representative emission source. Another difference between the two units was the maximum charge level of the main capacitor: $54 \mathrm{~J}$ for the $\mathrm{BB}$ unit versus $57 \mathrm{~J}$ for the flight unit. This difference was assumed to be insignificant. Testing at GRC has shown that the spark event is initiated in the spark plug when a certian threshold voltage is reached. To a first order approximation. this is independent of the maximum capacitur voltage. A limited sampling of the vacuum firing BB PPT emissions in the highest emission range. $200 \mathrm{KHz} 0+\mathrm{MHz}$. showed good agreement with results from the PPT flight unit. Athough a limited sample was taken because of time and equipment constraints, the BB PPT emissions were within the scatter of the flight unit emissions and the peath level was within 5 to $10 \mathrm{db}$ of the flight unit at mon frequencies. A sample of the ras lest datid for coteh PPT unt is shown in Figure $-T$. Tomblate the PPT spaceratt incertace a spate night PPT

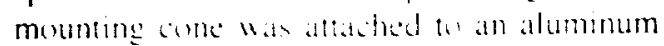
sheet that represented the spacerall pamel

\section{Configuration of Shield Validation Test}

The base plates of the PPT enclosure were attached to the mounting cone. as they would be when attached to the flight unit. An aluminum stand was placed on top of the base plates to allow the BB PPT to rest on a flat surface without constructing a complicated adapter to connect the BB PPT to the flight-mounting cone. The end of the mounting cone that would attach to the flight PPT electronics housing was capped off with a steel plate. The enclosure cover was placed over the BB PPT and attached to the base plates with fasteners. Care was taken to ensure that the BB PPT was grounded in the same manner as the flight unit. Copper tape was used to cover any open gaps between the base plates and the mounting cone. Copper tape was also used to minimize any gaps between the harnesses and the channel walls.

The test configuration is shown in the Figure 8 . The BB PPT is in the EMI enclosure that is mounted on the simulated spacecraft interface. The PPT GSE is located in the white shield box on the right. The PPT GSE used for ambient discharge was configured and powered on before sealing it in its shielding box prior to BB PPT firing. The EMI test antenna is located 1 meter away from the location of the spark plug and is in the direction of what would be the ALI location on the spacecraft. The command and telemetry GSE. as well as the power supply for the PPT unit. were located outside the test chamber. The power supply and command and telemetry GSE simulate the spacecraft bus interfaces to the PPT.

\section{Shield Validation Test Procedure and Results}

In order to compare the effectiveness of the shield. MIL-STD-461C RE02 tests were performed with the same adaptations used in the flight unit test program. The only significant difference between the tests was the number of samples taken at each selected frequency. Only ten samples were taken at each

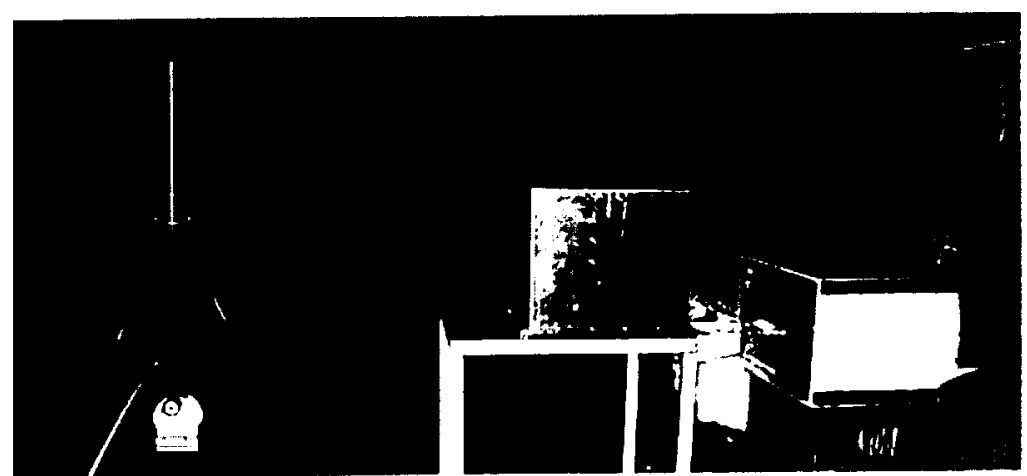

Figure 8. PPT EMI Shield Validation Test 
However. 10 samples were believed to be sufficient to represent the shields effectiveness.

The results from these tests are shown in Figure 9 Included in this figure are the EO- 1 component limit line for broadband emissions and the previous results from the flight PPT firing in vacuum.

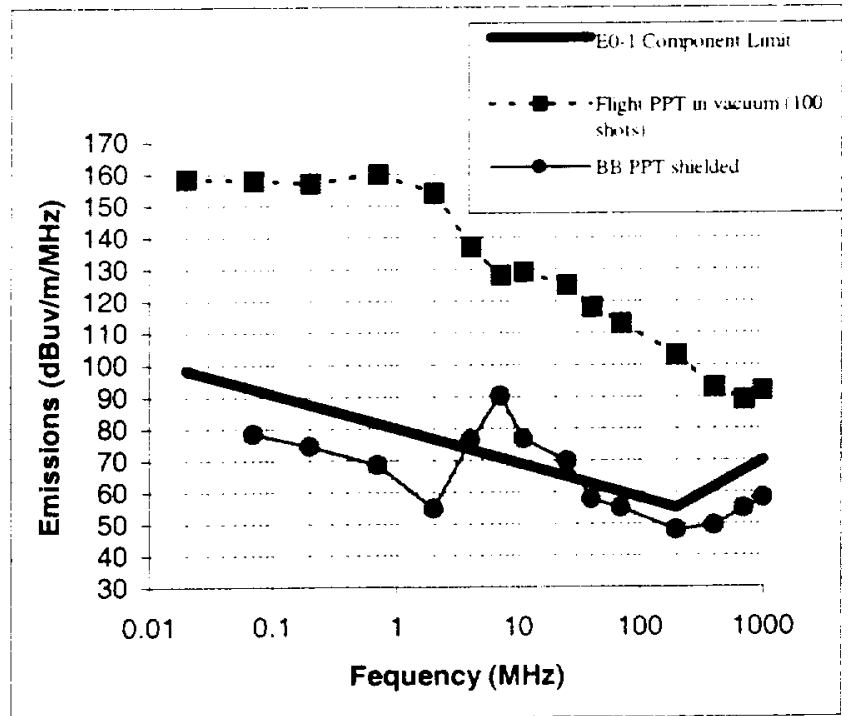

Figure 9: REO2 Results from PPT Shield Test

The emissions were significantly reduced across the entire frequency range. The emissions exceeded the EO-1 component limit line only between approximately 3.5 and $30 \mathrm{MHz}$ by a maximum of $20 \mathrm{db}$ at $7 \mathrm{MHz}$. These levels were determined to be acceptable because they fell within the measured emission values of the spacecraft operating with the ALI instrument functioning without the PPT. Spacecraft level RE02 broadband exceeded the specified spacecraft level limits by 5 to $10 \mathrm{db}$ between approximately 2 to 30 $\mathrm{MHz}$. The spacecraft level limits were $10 \mathrm{~dB}$ higher then the component level limits.

The raw data from BB PPT tests indicated that emission levels from the flight unit during ambient air discharge tests might be even lower because of the difference between the flight and BB PPT in the main capacitor charging circuit. For the frequencies that had the highest emissions. it was observed that the peaks occurred during the charge cycle of the BB main capacitor. The discharge events had a much lower peak emission value than the noise in the charge cycle at these frequencies. In reviewing previous data on both the flight unit and the $B B$ unit. it was observed that the BB PPT always had this characteristic while the flight unit did not

\section{Spacecraft Level Tests with PPT EMI shicld}

The PPT EMI shield was utilized on the spacecraft for two series of functional tests following spacecraft environmental testing. These tests included sending discrete fire commands to the PPT as well as closed loop autonomous commanding of the PPT by the EO-1 attitude control system. Hundreds of shots were accumulated over the entire range of PPT charge levels. The only anomalies to occur during these tests were associated with PPT GSE. These problems were corrected and the PPT and spacecraft performed as expected.

The successful on-orbit validation of all of EO-l's science instruments and other New Millennium Program technologies proved that the PPT ground test program did not damage any flight component.

\section{Conclusions}

A conservative approach was taken to insure that the radiated emissions from the EO-1 PPT did not adversely affect the Advanced Land Imager or other EO-1 subsystems: On-orbit tests of the PPT have been delayed until after the completion of the validation of all EO-1 instruments. To allow the PPT to be functionally tested at the spacecraft level before launch, an EMI shield was designed, constructed, and validated. The EMI shield was successfully implemented; allowing the PPT to be tested end-to-end at the spacecraft level while providing protection for the ALI. The on-orbit test of the PPT. scheduled for September/October 2001 should verify PPT compatibility with the entire E0-1 spacecraft.

This experience suggests the need for a standard in measuring and evaluating low repetition pulsed discharges from electric propulsion devices and determining the effects they may have on integrated circuits. The MIL-STD-461 tests were not targeted to address this uncommon emitter type. Previous programs have almost universally relied on test firing propulsion equipment with the actual flight units of engineering test units of the instrument or subsystem for which electromagnetic compatibility was of concern. Developmental instruments and subsystems. such as the ALI on EO-1, cannot always afford the risk to the flight unit or the cost of producing an engineering test unit. A new test method and evaluation criteria would enable spacecraft developers to better assess the risk of flying electric propulsion systems within program resource constraints 


\section{References}

1. Benson. S.W. Arrington. L.A.. Hoskins, W.A.. "Development of a PPT for the EO- 1 Spacecraft". AIAA-99-2276. June 1999.

2. Arrington, L.A.. Haag. T.W., "Multi-Axis Thrust Measurements of the EO-1 Pulsed Plasma Thruster", AIAA 99-2290, June 1999.

3. Hoskins, W.A., Wilson. M.J., Willey, M.J., Meckel, N.J.. Campbell. M. Chung, S., "PPT Development Efforts at Primex Aerospace Company". AIAA 99-2291. June 1999.

4. Meckel, N.J.. Cassady, R.J., Osborne, R.D. Hoskins, W.A., and Myers. R.M., "Investigation of Pulsed Plasma Thrusters for Spacecraft Attitude Control, " IEPC-97-128, Au. 1997.

5. Cassady, R.J., Morris, J.P., Vaughan, C.E., Willey, M.J., "New Attitude Control Strategies Using Pulsed Plasma Thruster Systems," AAS 98-065, February, 1998

6. Bicknell, W.E., Constantine. J.D., Forman, S.E., Lencioni. D.E., "EO-1 Advanced Land Imager," SPIE, San Diego, July, 1999.

7. Javor, K. "Derivation of Radiated Emission Limits," Electronic Industries Association Meeting \# 72, 28-29 March, 1990.

8. Begun, M., Guman, W.J., "Pulsed Plasma Radio Frequency Interference Studies", AFRPL-TR-77-85. September. 1977.

9. NASA/GFSFC Document 5493-106-99. "Electromagnetic Compatibility (EMC) Test Procedure For The Earth Orbiter-1 (EO-1) Pulsed Plasma Thruster (PPT)

10. M.J.Cully, C. Gay, J. Thurber, "Small Spacecraft Bus Development in the New Millennium" 12th AIAA / USU Conference onSmall Satellites, September 1998 